\title{
FRACTIONAL ITERATION OF ENTIRE AND RATIONAL FUNGTIONS
}

\author{
G. SZEKERES
}

(received 2 July 1963)

\section{Introduction}

We shall be concerned with the behaviour of the fractional iterates of analytic functions which have a fixpoint $\zeta$ with multiplier $1^{1}$. The general form of such a function is

$$
f(z)=z+\sum_{k=m+1}^{\infty} a_{k}(z-\zeta)^{k}, \quad a_{m+1} \neq 0 \quad(m \geqq 1)
$$

if $\zeta$ is finite,

$$
f(z)=z+\sum_{k=m-1}^{\infty} a_{k} z^{-k}, \quad a_{m-1} \neq 0 \quad(m \geqq 1)
$$

if $\zeta=\infty$.

Given a formal power series of the form $(1)$ or $\left(1^{*}\right)$ we can determine for every real or complex $s$ a unique formal power series

$$
f_{s}(z)=z+\sum_{k=m+1}^{\infty} a_{k}^{(s)}(z-\zeta)^{k}, \quad \quad a_{m+1}^{(s)}=s a_{m+1}
$$

or

$$
f_{s}(z)=z+\sum_{k=m-1}^{\infty} a_{k}^{(s)} z^{-k}, \quad a_{m-1}^{(s)}=s a_{m-1}
$$

from the formal identities

$$
f \circ f_{8}(z)=f_{8} \circ f(z)
$$

(where the notation $f \circ g(z)$ stands for $f(g(z)))$.

If $s=n$ is a non-negative integer then $f_{n}(z)$ is the $n$-the iterate of $f(z)$,

$$
f_{0}(z)=z, \quad f_{n+1}(z)=f \circ f_{n}(z) \quad(n \geqq 0) .
$$

and $f_{-1}(z)$ is the formal inverse of $f(z)$,

$1 \zeta$ is a fixpoint of $f(z)$, with multiplier $\mu$, if $f(\zeta)=\zeta$ and $f^{\prime}(\zeta)=\mu$ if $\zeta$ is finite, $f^{\prime}(\zeta)=1 / \mu$ if $\zeta=\infty$. 


$$
f \circ f_{-1}(z)=f_{-1} \circ f(z)=z \text {. }
$$

Generally, the $f_{s}(z)$ form a family of (real or complex) fractional iterates of $f(z)$, satisfying the equations

$$
f_{s} \circ f_{t}(z)=f_{t} \circ f_{s}(z)=f_{t+t}(z), \quad f_{1}(z)=f(z),
$$

for every $s, t$. (Baker [2], p. 268).

Suppose now that the series (1) or $\left(1^{*}\right)$ converges $^{2}$; then so do all iterates $f_{n}(z)$ for integral $n$. For arbitrary complex $s, f_{8}(z)$ may or may not converge; for example if

$$
f(z)=e^{x}-1=z+\frac{1}{2} z^{2}+\cdots
$$

then $f_{s}(z)$ converges only for integral values of $s$ (Baker $[1]$, p. 160). On the other hand if

$$
f(z)=z /(1+z)=z-z^{2}+z^{3}-\cdots
$$

then each

$$
f_{s}(z)=z /(1+s z)=z-s z^{2}+s^{2} z^{3}-\cdots
$$

converges $^{3}$; generally if

$$
(z-\zeta) a(z)=1+b_{1}(z-\zeta)+b_{2}(z-\zeta)^{2}+\cdots
$$

is holomorphic at $z=\zeta$ (hence

$$
a_{-1}(w)=\zeta+w^{-1}+b_{1} w^{-2}+c_{3} w^{-3}+\cdots
$$

is holomorphic at $w=\infty$ ) then

$$
f_{s}(z)=a_{-1}(a(z)+s)=z-s(z-\zeta)^{2}+a_{3}(z-\zeta)^{3}+\cdots
$$

is holomorphic at $z=\zeta$ and the $f_{s}(z)$ form a family of fractional iterates at the fixpoint $\zeta$.

These examples raise the question, which of the two types of behaviour, exemplified by $z /(1+z)$ or $e^{z}-1$, is the more typical one for entire and rational functions. The main objective of the present paper is to find an answer to this question. Somewhat unexpectedly, we shall find that all entire transcendental functions behave like $e^{z}-1$, and among rational and entire rational functions the bilinear and linear ones are the only exceptions.

THEOREM 1. Suppose that $f(z)$ is a rational or entire function which has a fixpoint $\zeta$ (finte or infinite) with multiplier 1. Suppose furthermore that $f(z)$ has a family of fractional iterates $f_{s}(z)$ with the property that for every real $\sigma$,

\footnotetext{
For brevity we say that a series of the form (1) or $\left(1^{*}\right)$ converges if it converges for some finite non-zero value of $z$.

A more trivial example is $f(z)=z+1, f_{s}(z)=z+s$.
} 
$f_{\sigma}(z)$ is holomorphic at $z=\zeta$. Then $f(z)$ is a bilinear function of the form

$$
f(z)=\zeta+(z-\zeta) /(1+a(z-\zeta))
$$

with

$$
f_{s}(z)=\zeta+(z-\zeta) /(1+a s(z-\zeta))
$$

or a linear function

$$
f(z)=z+a
$$

with $f_{0}(z)=z+$ fas.

Otherwise stated, if $f(z)$ is rational or entire and not of the form (7) or (8) then there is a positive integer $p$ with the property that (for real $s$ ) the formal fractional iterates (2), $\left(2^{*}\right)$ converge if and only if $s$ is an integral multiple of $1 / p^{4}$. For, by a theorem of Baker ([2], p. 285) the set of real $\sigma$ for which $f_{\sigma}(z)$ converges is either the whole real line or a discrete lattice on the real line.

Theorem 1 considerably extends the following result of Baker ([2], p. 292): If $f(z)$ is a non-linear entire function of the form (1) then there can only be countably many $s$ for which the fractional iterates (2) are entire functions.

Whether Theorem 1 remains true for meromorphic functions (or more generally for single valued analytic functions) is an open question; I know of no counterexample ${ }^{5}$. For algebraic functions the theorem is certainly not valid. A counter-example is

where

$$
w_{s}=f_{s}(z)=z-s z^{2}+\cdots
$$

$$
z w^{2}-w_{s}\left(1+z^{2}+s z\right)+z=0 .
$$

The $f_{s}(z)$ form a family of holomorphic iterates at $z=0$ (they are in fact derived by (6) from $a(z)=z+z^{-1}$ ) and each member of the family is an algebraic function of degree 2.

The proof of Theorem 1 will be based on a study of iteration orbits and continuation of $f_{s}(z)$ along such orbits. The possibility of such an approach was suggested to me several years ago by C. A. B. Smith who used a similar (but partly heuristic) argument to show that the fractional iterates of $z+z^{2}$ cannot possibly converge at 0 for all values of $s^{6}$.

- It is not known whether it is possible for the series (2) to converge when $s$ is not real and $f(z)$ is not of the form (7) or (8).

- Note added in proof. Since this paper was written, I. N. Baker has extended the validity of Theorem 1 to meromorphic functions. For single-valued function in general, the question is still open.

- Oral communication. 
In formulating the ideas of the proof I was greatly helped by numerical experiments on iteration orbits of various entire functions, carried out by K. W. Mills on the IBM 1620 computer at the University of Adelaide.

\section{Preliminaries. The case $m=1$}

For the study of iteration orbits it is convenient to assume that the fixpoint is situated at $\infty$. We can always displace a finite fixpoint $\zeta$ to infinity by the transformation

$$
f^{*}(z)=\Phi^{*} f(z)=1 /\left(f\left(z^{-1}+\zeta\right)-\zeta\right)
$$

If $f(z)$ has an expansion of the form (1) at $\zeta$ then $f^{*}(z)$ will have an expansion

$$
f^{*}(z)=z+\sum_{k=m-1}^{\infty} a_{k}^{*} z^{-k}, \quad a_{m-1}^{*}=-a_{m-1}
$$

at $\infty$. By a subsequent transformation

where

$$
\Phi * * *(z),=b^{-1} f^{*}(b z)=z+\sum_{k=m-1}^{\infty} a_{k}^{* *} z^{-k},
$$

$$
b^{m}=a_{m-1}^{*}
$$

we can achieve that $a_{m-1}^{* *}=1$. Hence $g(z)=\Phi f(z)=\Phi^{* *} \Phi^{*} f(z)$ has the normal form

$$
g(z)=z+z^{-m+1}+\sum_{k=m}^{\infty} a_{k} z^{-k}
$$

where the asterisks have been dropped from $a_{k}^{* *}$. Note that if $f_{s}(z)$ is holomorphic at $z=\zeta$ and $g_{s}(z)=\Phi f_{s}(z)$, then

$$
g_{s}(z)-z=s z^{-m+1}+\sum_{k=m}^{\infty} a_{k}^{(\bullet)} z^{-k}
$$

is holomorphic at $z=\infty$. Furthermore if the $f_{s}(z)$ form a family of fractional iterates of $f(z)$ then the $g_{s}(z)$ form a family of fractional iterates of $g(z)$. In fact $\Phi f(z)$ has the form $\phi^{-1} \circ f \circ \phi(z)$ where $\phi(z)$ is a bilinear transformation. Therefore it is sufficient to prove Theorem 1 in the following form:

THEOREM 1*. Let $g(z)$ be single valued and meromorphic over the extended complex plane with the possible exception of $z=0$ where it may have an essential singularity; in the latter case we assume that $g(z) \neq 0$ for $z \neq 0$. Suppose further that $g(z)$ has a convergent expansion

$$
g(z)=z+z^{-m+1}+\sum_{k=m}^{\infty} a_{k} z^{-k}
$$


at $z=\infty$ for some $m>0$. Let the series

$$
g_{s}(z)=z+s z^{-m+1}+\sum_{k=1 / 2}^{\infty} a_{k}^{(s)} z^{-k}
$$

be determined from

$$
g \circ g_{s}(z)=g_{s} \circ g(z)
$$

and suppose that $g_{\sigma}(z)$ converges for every real $\sigma$. Then $g(z)=z+1$.

For convenience we shall assume at first that $m=1$, i.e.

$$
\begin{gathered}
g(z)=z+1+\sum_{k=1}^{\infty} a_{k} z^{-k}, \\
g_{\triangleleft}(z)=z+s+\sum_{k=1}^{\infty} a_{k}^{(z)} z^{-k} .
\end{gathered}
$$

The necessary modifications when $m>1$ are of a fairly trivial (though bothersome) character and will be indicated in the last section.

Determine the formal series

$$
b(z)=1-a_{1} z^{-1}+\cdot \sum_{k=2}^{\infty} b_{k} z^{-k}
$$

from

$$
b \circ g(z)=b(z) / g^{\prime}(z) .
$$

Clearly $b(z)=a^{\prime}(z)$ where

$$
a(z)=z-a_{1} \log z-\sum_{k=1}^{\infty} k^{-1} b_{k+1} z^{-k}
$$

is a formal solution of Abel's equation

$$
a \circ g(z)=a(z)+1, \quad a \circ g_{8}(z)=a(z)+s .
$$

Our first aim is to prove that the series (13) for $b(z)$ converges for large $z$. The necessary tools were developed by I. N. Baker in [2] and we shall rely heavily on his work.

Given $K>0$ and $|\eta|=1$ ( $\eta$ complex) we define the sets

$$
\begin{gathered}
\mathscr{S}(\eta, K)=\left\{z \mid \operatorname{Re}\left(z \eta^{-1}\right)>K\right\}, \\
\mathfrak{D}(\eta, K)=\bigcup_{-\pi / 4 \leqq \theta \leqq \pi / 4} E\left(\eta e^{i \theta}, K\right), \\
\Im(\eta)=\left\{s \mid \eta^{-1} s=\sigma+i \tau, \sigma \geqq 0,-\sigma \leqq \tau \leqq \sigma\right\} .
\end{gathered}
$$

LEMmA 1. Suppose that the series (11) and (12) for $g(z)$ and $g_{-1}(z)$ converge for $|z|>R(>0)$. Then there is a $K_{0} \geqq R$ such that for all $K \geqq K_{0}$ the following is true: 
1. $z \in \mathfrak{D}(1, K)$ implies $g(z) \in \mathfrak{D}(1, K)$ and $\lim _{n \rightarrow \infty} g_{n}(z)=\infty$. $z \in \mathfrak{D}(-1, K)$ implies $g_{-1}(z) \in \mathscr{D}(-1, K)$ and $\lim _{n \rightarrow \infty} g_{-n}(z)=\infty$.

2. There is a function $a^{(+)}(z)$, holomorphic and schlicht in $\mathfrak{D}(1, K)$, satisfying $a^{(+)} \circ g(z)=a^{(+)}(z)+1$.

There is a function $a^{(-)}(z)$, holomorphic and schlicht in $\mathfrak{D}(-1, K)$, satisfying $a^{(-)} \circ g_{-1}(z)=a^{(-)}(z)-1$.

Furthermore

$$
b^{(+)}(z)=\frac{d}{d z} a^{(+)}(z) \rightarrow 1 \text { uniformly as } z \rightarrow \infty \text { in } \mathfrak{D}(1, K) .
$$

$\left(20^{*}\right) \quad b^{(-)}(z)=\frac{d}{d z} a^{(-)}(z) \rightarrow 1$ uniformly as $z \rightarrow \infty$ in $\mathfrak{D}(-1, K)$.

3. $b^{(+)}(z)$ and $b^{(-)}(z)$ are uniquely determined by conditions (20) and (20*).

Statements 1 and 2 follow immediately from Baker's lemmas 2 and 3 in [2]. To prove 3, we note that $b(z)=b^{(+)}(z)$ satisfies equation (14). Hence if $b^{*}(z)$ is a second function satisfying equation $(14)$ in $\mathfrak{D}(1, K)$ then

$$
b^{*} \circ g(z) / b \circ g(z)=b^{*}(z) / b(z) \text {. }
$$

Suppose that for some $z \in \mathfrak{D}(1, K), b^{*}(z)=\lambda b(z)$, then by (21) $b^{*} \circ g_{n}(z)=\lambda b \circ g_{n}(z)$ for every $n \geqq 0$ hence

$$
\lim _{n \rightarrow \infty} b^{*} \circ g_{n}(z)=\lambda \lim _{n \rightarrow \infty} b \circ g_{n}(z)=\lambda
$$

by properties 1 and 2 . Hence $\lambda=1, b^{*}(z)=b(z)$, provided that $b^{*}(z)$ satisfies condition (20). The proof of uniqueness of $b^{(-)}(z)$ is similar.

LEMMA 2. Suppose that $a(z)$ is holomorphic and schlicht in $\mathbb{E}\left(\eta, K_{0}\right)$ for some $K_{0}>0,|\eta|=1$, and

$$
b(z)=a^{\prime}(z) \rightarrow 1 \text { uniformly as } z \rightarrow \infty \text { in } \&\left(\eta, K_{0}\right) .
$$

Then there is a $K_{1} \geqq K_{0}+2$ such that for every $K \geqq K_{1}$ and $z_{0} \in \mathbb{E}(\eta, K)$ the equation

$$
a\left(z_{s}\right)=a\left(z_{0}\right)+s
$$

has a unique solution $z_{s} \in \mathfrak{S}(\eta, K)$ if $|s| \leqq 1$ or $s \in \mathfrak{S}(\eta)$.

Furthermore for given $|s| \leqq 1$ or $s \in \mathbb{S}(\eta)$.

$$
\lim \left(z_{s}-z_{0}\right)=s \text { uniformly as } z_{0} \rightarrow \infty \text { in } \mathbb{E}(\eta, K),
$$

while for given $z_{0} \in \mathfrak{E}(\eta, K)$ and $s \in \mathfrak{S}(\eta)$

$$
\lim z_{n:}=\infty \text {. }
$$


Proof. We may assume that $\eta=1$; for otherwise write $z=\eta z^{*}$, $a(z)=\eta a^{*}\left(z^{*}\right), s=\eta s^{*}$ and prove the statement for the starred quantities. Note that

$$
b(z)=\frac{d}{d z} a(z)=\frac{d}{d z^{*}} a^{*}\left(z^{*}\right)=b^{*}\left(z^{*}\right) \rightarrow 1 \text { as } z^{*} \rightarrow \infty \text { in }\left(E\left(1, K_{0}\right) .\right.
$$

Now given $N \geqq 1$, let $R_{1}=R_{1}(N) \geqq K_{0}$ be so large that $|b(z)-1|<\frac{1}{5} N$ for $z \in \mathbb{E}\left(1, K_{0}\right),|z|>R_{1}$. Such an $R_{1}$ exists, because of (22). By definition of the set $\mathfrak{S}(1)$ we have $\left|z_{0}+s+h\right|>R_{1}$ for every $z_{0} \in \mathbb{E}\left(1, K_{0}+2\right)$, $\left|z_{0}\right|>R_{1}+2,|h| \leqq \frac{1}{2}$ and $s \in \subseteq(1)$ or $|s| \leqq 1$.

Suppose that $z_{0} \in \mathbb{E}(1, K+2),\left|z_{0}\right|>R_{1}+2$, where $K \geqq K_{0}$, and let $|h|=|s| / 2 N$. Then writing $s=\sigma+\tau i, \operatorname{Re}\left(z_{0}+s+h\right)>K+2+\sigma-\frac{1}{2}|s|>K$ if $|s| \leqq 1$ or $s \in \mathfrak{S}(1)$, hence $z_{0}+s+h \in \mathbb{E}(1, K)$ and

$$
\begin{aligned}
& a\left(z_{0}+s+h\right)-a\left(z_{0}\right)=\int_{z_{0}}^{s_{0}+s+h} b(\zeta) d \zeta=(s+h)(1+\gamma),|\gamma|<1 / 5 N, \\
& \left|a\left(z_{0}+s+h\right)-a\left(z_{0}\right)-s\right| \geqq(1-|\gamma|)|h|-|s \gamma|>\frac{4}{5} \frac{1}{2 N}|s|-\frac{1}{5 N}|s|=|s| / 5 N, \\
& \left|a\left(z_{0}+s\right)-a\left(z_{0}\right)-s\right| \leqq|s \gamma|<|s| / 5 N .
\end{aligned}
$$

Hence $a\left(z_{s}\right)-a\left(z_{0}\right)-s=0$ has a solution $z_{s}$ with

$$
\left|z_{s}-z_{0}-s\right|<|s| / 2 N
$$

i.e. with $z_{\varepsilon} \in \mathbb{E}(1, K)$. This solution is unique because of the schlichtness of $a(z)$ in $\mathbb{E}\left(1, K_{0}\right)$. By setting $K_{1}=R_{1}(1)+2$, the first statement of the lemma is obtained. The validity of (24) and (25) follows from the fact that $N$ in (26) can be chosen arbitrarily large.

Inequality (26) gives, when applied to $z_{n}$ instead of $z_{0},\left|z_{n+1}-z_{n}-1\right|<1 / 5 N$, where $N$ can be taken as large as we please, provided that $n$ is sufficiently large. Hence writing

$$
z_{n}=z_{n-1}+1+\rho_{n}, \quad n=1,2, \cdots
$$

we obtain $\rho_{n} \rightarrow 0$ as $n \rightarrow \infty$. Or, if $z_{n}=x_{n}+i y_{n}$, we get $x_{n} / y_{n} \rightarrow 0$ as $n \rightarrow \infty$. This gives

Lemma 3. Let $a(z), K_{1}$ be as in Lemma $2,|\eta|=1, s \in \mathbb{S}(\eta), z_{0} \in \mathbb{E}\left(\eta, K_{1}\right)$ and

$$
\eta^{-1} z_{\sigma}=x_{\sigma}+i y_{\sigma},
$$$$
\sigma \geqq 0
$$

where $z_{\sigma}$ is a solution of $a\left(z_{\sigma}\right)=a\left(z_{0}\right)+\sigma$. Then

$$
\lim _{\sigma \rightarrow \infty} y_{\sigma} / x_{\sigma}=0 \text {. }
$$

By applying Lemma 2 (with $\eta=1$ ) to Lemma 1 we find that for real 
$\sigma \geqq-1$ there is a uniquely determined holomorphic function $g_{\sigma}^{(+)}(z)$ in $\mathfrak{D}\left(1, K_{1}\right)$ satisfying

$$
\begin{gathered}
a^{(+)} \circ g_{\sigma}^{(+)}(z)=a^{(+)}(z)+\sigma, \\
\lim \left(g_{\sigma}^{(+)}(z)-z\right)=\sigma \text { when } z \rightarrow \infty \text { in } \mathfrak{D}\left(1, K_{1}\right) .
\end{gathered}
$$

In particular if $z_{n}$ denotes $g_{n}(z)$ then

$$
\lim _{n \rightarrow \infty}\left(g_{\sigma}^{(+)}\left(z_{n}\right)-z_{n}\right)=\sigma
$$

by property 1 of Lemma 1 . But $g_{\sigma}^{(+)} \circ g=g \circ g_{\sigma}^{(+)}$, therefore

$$
\lim _{n \rightarrow \infty}\left\{g_{n}\left(g_{\sigma}^{(+)}(z)\right)-g_{n}(z)\right\}=\sigma .
$$

The same relation, namely

$$
\lim _{n \rightarrow \infty}\left\{g_{n}\left(g^{*}(z)\right)-g_{n}(z)\right\}=\sigma(\geqq-1)
$$

holds for any function $g^{*}(z)$, holomorphic in $\mathfrak{D}(1, K)$, which satisfies the conditions

$$
\lim \left(g^{*}(z)-z\right)=\sigma \text { when } z \rightarrow \infty \text { in } \mathfrak{D}\left(1, K_{1}\right)
$$

and $g \circ g^{*}=g^{*} \circ g$. Since for given $z, z^{*} \in \mathfrak{D}\left(1, K_{0}\right), s$ is uniquely determined from the equation $s=a^{(+)}\left(z^{*}\right)-a^{(+)}(z)$,

$$
\lim _{n \rightarrow \infty}\left\{g_{n}\left(z^{*}\right)-g_{n}(z)\right\}=\sigma
$$

implies $z^{*}=g_{\sigma}^{(+)}(z)$. Hence we conclude:

LEMMA 4. Suppose that $g^{*}(z)$ is holomorphic in $\mathfrak{D}(1, K)$ for some $K \geqq K_{1}$ and $g \circ g^{*}=g^{*} \circ g$. Suppose further that

$$
\lim \left(g^{*}(z)-z\right)=\sigma(\geqq-1) \text { when } z \rightarrow \infty \text { in } \mathfrak{D}(1, K) .
$$

Then $g^{*}=g_{\sigma}^{(+)}$.

Similarly by applying Lemma 2 with $\eta=-1$ to Lemma 1 we obtain a unique holomorphic $g_{-\sigma}^{(-)}(z)$ in $\mathfrak{D}\left(-1, K_{1}\right)$ for $\sigma \geqq-1$ satisfying $a^{(-)} \circ g_{-\sigma}^{(-)}(z)=a^{(-)}(z)-\sigma$,

$$
\lim \left(g_{-\sigma}^{(-)}(z)-z\right)=-\sigma \text { when } z \rightarrow \infty \text { in } \mathfrak{D}\left(-1, K_{1}\right) .
$$

As before, we find

LEMMA 4*. Suppose that $g^{*}(z)$ is holomorphic in $\mathfrak{D}(-1, K)$ for some $K \geqq K_{1}, g \circ g^{*}=g^{*} \circ g$, and

$$
\lim \left(g^{*}(z)-z\right)=-\sigma(\leqq 1) \text { when } z \rightarrow \infty \text { in } \mathfrak{D}(-1, K) .
$$

Then $g^{*}=g_{-\sigma}^{(-)}$. 
As a corollary we obtain: if the series (12) for $g_{\sigma}(z)$ (where $-1 \leqq \sigma \leqq 1$ ) converges for sufficiently large $z$ then it is identical with $g_{\sigma}^{(+)}(z)$ and $g_{\sigma}^{(-)}(z)$. Hence we conclude:

Lemma 5. If the series (12) for $g_{\sigma}(z)$ (where $-1 \leqq \sigma \leqq 1$ ) converges for sufficiently large $z$ then the function represented by the series is holomorphic in

$$
\mathfrak{D}(K)=\mathfrak{D}(1, K) \cup \mathfrak{D}(-1, K)
$$

where $K$ is a suitable positive number, independent of $\sigma$.

This result is essentially contained in Baker's Theorem 7 in [2]. In particular if every series $g_{\sigma}(z),-1 \leqq \sigma \leqq 1$ converges then they represent a holomorphic family of iterates of $g(z)$ in $\mathfrak{D}(K)$, identical with $g_{\sigma}^{(+)}(z)$ and $g_{\sigma}^{(-)}(z)$ in $\mathfrak{D}(1, K)$ and $\mathfrak{D}(-1, K)$ respectively. Since the family $g_{\sigma}(z)$ uniquely determines $b(z)$, we conclude that the direct continuation of $b^{(+)}(z)$ from $\mathfrak{D}(1, K)$ to $\mathfrak{D}(-1, K)$ is identical with $b^{(-)}(z)$. Hence by property 2 of Lemma $1, b(z)$ is holomorphic at $O$ and the series $(13)$ for $b(z)$ converges for large $z$. Thus we obtain

THEOREM 2. Suppose that each of the series (12) converges for large $z$ when $s=\sigma,-1 \leqq \sigma \leqq 1$; then there exists an $R_{1}>0$ such that the series (13) for $b(z)$ and each of the series (12) for $g_{s}(z)(|s| \leqq 1)$ converges for $|z|>R_{1}$.

In particular (12) converges for every complex $s$.

Henceforth we assume that each series (12) (hence also the series (13) for $\left.b(z)=a^{\prime}(z)\right)$ converges. We shall call the functions represented by these series the regular branches of $g_{s}(z), b(z)$. For every $z_{0} \in \mathfrak{D}\left(-1, K_{0}\right)$ where $K_{0}$ is as in Lemma 1, we can define an iteration orbit through $z_{0}$,

$$
\mathfrak{L}\left(z_{0}\right): \quad z=\psi\left(z_{0}, \sigma\right) \quad-\infty<\sigma<\infty
$$

as follows:

$$
\begin{array}{ll}
\psi\left(z_{0}, \sigma\right)=g_{\sigma}\left(z_{0}\right) & \text { for } \sigma \leqq 0, \\
\psi\left(z_{0}, \sigma\right)=g\left(\psi\left(z_{0}, \sigma-1\right)\right) & \text { for } \sigma>0,
\end{array}
$$

where $g_{\sigma}$ denotes the regular branch of the function. In particular $\varphi\left(z_{0}, 0\right)=z_{0}$. The definition is valid because if $g(z)$ is not rational (hence $g(z)$ is undefined for $z=0)$ then $g(z) \neq 0$ for $z \neq 0$ and hence $\psi\left(z_{0}, \sigma\right) \neq 0$. Note that $\left(28^{*}\right)$ is valid for every real $\sigma$.

Suppose now that $z_{0}=i t_{0}$ where $t_{0}$ is sufficiently large, then by Lemma 2 , $g_{\sigma}\left(z_{0}\right) \in \mathfrak{D}\left(1, K_{0}\right) \cap \mathfrak{D}\left(-1, K_{0}\right)$ for $-1 \leqq \sigma \leqq 1$ hence by Lemma 1

$$
g_{\sigma+n}\left(z_{0}\right) \in \mathfrak{D}\left(1, K_{0}\right), \quad g_{\sigma-n} \in \mathfrak{D}\left(-1, K_{0}\right)
$$

for $n=1,2, \cdots$, and $\psi\left(z_{0}, \sigma\right) \in \mathfrak{D}\left(K_{0}\right)=\mathscr{D}\left(1, K_{0}\right) \cup \mathfrak{D}\left(-1, K_{0}\right)$ for every real $\sigma$. Thus $\mathfrak{L}\left(z_{\mathbf{0}}\right)$ has the following properties: 


$$
\psi\left(z_{0}, \sigma\right) \in \mathscr{D}\left(1, K_{0}\right), \psi\left(z_{0},-\sigma\right) \in \mathfrak{D}\left(-1, K_{0}\right) \text { for } \sigma \geqq \sigma_{0}
$$

where $\sigma_{0}$ is a sufficiently large non-negative number (actually in the present case we can take $\sigma_{0}=0$ ),

$$
\psi\left(z_{0}, \sigma_{0}+\delta\right)=g_{\sigma}\left(z_{0}, \sigma_{0}\right) \quad 0 \leqq \delta \leqq 1
$$

where $g_{\sigma}$ is the regular branch of the function.

A path of the form (27), (28), $\left(2^{*}\right)$ with properties (29) and (30) will be called a regular iteration orbit. It follows from (14) that $b(z)$ has a holomorphic continuation from $\mathfrak{D}\left(1, K_{0}\right)$ into $\mathfrak{D}\left(-1, K_{0}\right)$ along $-\mathfrak{Q}\left(z_{0}\right)$, and in fact the continuation results in the regular branch of $b(z)$. For

$$
\int_{z_{0}}^{\psi\left(z_{0}, \sigma\right)} b(\zeta) d \zeta=\sigma
$$

by (16), (28) and $\left(28^{*}\right)$, hence

$$
b\left(\psi\left(z_{0}, \sigma\right)\right)=1 / \frac{\partial}{\partial \sigma} \psi\left(z_{0}, \sigma\right)
$$

along $\mathfrak{L}\left(z_{0}\right)$, from which the statement follows by (28). We show that

$$
\begin{aligned}
b(z) & \neq 0 \\
g^{\prime}(z) & \neq 0, \quad g^{\prime}(z) \neq \infty
\end{aligned}
$$

for $z \in \mathfrak{L}\left(z_{0}\right)$. For simplicity let us write $\psi(\sigma)$ for $\psi\left(z_{0}, \sigma\right)$. Suppose that $g(\psi(\sigma))=\psi(\sigma+1)=\infty$ for some $\sigma$; then $\psi(\sigma+2)=g(\psi(\sigma+1))=\infty$, $\psi(\sigma+n)=\infty$ for $n=1,2,3, \cdots$, contrary to $(29)$. Hence also $g^{\prime}(\psi(\sigma)) \neq \infty$. But by (14) $b(\psi(\sigma+1))=b(g(\psi(\sigma)))=b(\psi(\sigma)) / g^{\prime}(\psi(\sigma))$, therefore $b(\psi(\sigma+1))=0$ implies $b(\psi(\sigma))=0$ i.e. $b(\psi(\sigma-n))=0, n=0,1,2, \cdots$, contrary to (29) and property 2 of Lemma 1 . This proves (31). Finally if we had $g^{\prime}(\psi(\sigma))=0$ then by (14) and (31) we had $b(\psi(\sigma+1))=\infty$ and $b(\psi(\sigma+n))=\infty$ for $n=1,2, \cdots$, contrary to property 2 of Lemma 1 . Hence $\left(31^{*}\right)$ is true.

Denote by $\mathfrak{L}_{0}$ the iteration orbit $\mathfrak{L}\left(z_{0}\right)$ where $z_{0}=i t_{0}$ is fixed and chosen as above. $\mathfrak{\Omega}_{0}$ is a simple closed Jordan curve of the extended complex plane. For $\psi(\sigma)=\psi\left(\sigma^{\prime}\right), \sigma<\sigma^{\prime}$ implies

$$
\begin{gathered}
\psi(n+\sigma)=g_{n}(\psi(\sigma))=g_{n}\left(\psi\left(\sigma^{\prime}\right)\right)=\psi\left(n+\sigma^{\prime}\right), \\
g_{\sigma^{\prime}-\sigma}(\psi(n))=\psi(n) \text { for } n=1,2, \cdots
\end{gathered}
$$

which is impossible since $g_{\sigma^{\prime}-\sigma}(z)$ is holomorphic (and not a constant) at $\infty$ and $\psi(n) \rightarrow \infty$. Hence $\mathfrak{I}_{0}$ is simple, and also closed since $\psi(-n) \rightarrow \infty$, $\psi(n) \rightarrow \infty$, when $n \rightarrow \infty$.

Let $\mathfrak{S}_{0}$ denote the domain bounder from below by $\mathfrak{\Omega}_{0}$. $\mathscr{S}_{0}$ consists of all points 


$$
\psi(s)=g_{s}\left(z_{0}\right), \quad s=\sigma+\tau i, \quad-\infty<\sigma<\infty, \quad \tau>0 .
$$

In fact by (31), $a(\psi(s))-a\left(z_{0}\right)$ establishes a one to one conformal mapping of $\mathscr{G}_{0}$ onto the upper half $s$-plane $\tau>0$ through the equation

$$
a \circ \psi(s)=a \circ \psi(0)+s .
$$

In particular every path

$$
\mathfrak{S}_{\tau}: z=\psi(\sigma+\tau i)=g_{\sigma+\tau i}\left(z_{0}\right), \quad-\infty<\sigma<\infty
$$

where $\tau>0$, is a regular iteration orbit of $g(z)$.

From Lemma 3 (with $\eta=-1$ ) it follows that if $\sigma_{0}$ is a sufficiently large positive number then $\psi(-\sigma) \in \mathbb{E}\left(\eta_{0}, K_{1}\right)$ for $\sigma>\sigma_{0}$ where $\eta_{0}=e^{5 \pi i / 4}$. Similarly $\psi(\sigma) \in \mathbb{E}\left(\eta_{1}, K_{1}\right)$ for $\sigma>\sigma_{0}$ where $\eta_{1}=e^{-\pi i / 4}$.

By Lemma 2 (with $\eta=\eta_{0}$ ),

$$
g_{-\sigma_{0}-i \tau}\left(z_{0}\right)=\psi\left(-\sigma_{0}-i \tau\right) \in \mathbb{E}\left(\eta_{0}, K_{1}\right)
$$

and hence

$$
\psi(\sigma+i \tau) \in \mathfrak{D}\left(-1, K_{0}\right) \text { for } \sigma \leqq \sigma_{0}, \tau \leqq 0
$$

Similarly

$$
\psi(\sigma+i \tau) \in \mathfrak{D}\left(1, K_{0}\right) \quad \text { for } \quad \sigma \geqq \sigma_{0}, \tau \leqq 0 .
$$

We can now extend the definition of the iteration orbits $\mathfrak{I}_{\tau}$ for $\tau<0$ as follows:

$$
\begin{array}{ll}
\mathfrak{Q}_{\tau}: z=\psi(\sigma+i \tau), & -\infty<\sigma<\infty, \\
\psi(\sigma+i \tau)=g_{\sigma+i \tau}\left(z_{0}\right) & \text { for } \sigma \leqq-\sigma_{0} \\
\psi(\sigma+i \tau)=g(\psi(\sigma-1+i \tau)) & \text { for } \sigma \geqq-\sigma_{0} .
\end{array}
$$

By the same proof which showed that $\mathfrak{\Omega}_{0}$ is a regular iteration orbit it is seen that if $\tau_{\mathbf{1}}$ is sufficiently large then $\mathfrak{L}_{\boldsymbol{\tau}}$ is a regular iteration orbit for $\tau<-\tau_{1}$, and the points $z \in \mathfrak{R}_{\tau}, \tau<-\tau_{1}$ fill out a simply connected domain $B_{\tau_{1}}^{*}$ which is bounded from above by $\mathfrak{L}_{-\tau_{1}}$.

Let $\mathfrak{I}$ denote the set of all real $\tau$ with the following property $\tau \in \mathfrak{I}$ if and only if $\mathfrak{L}_{\tau^{\prime}}$ is a regular iteration orbit for every $\tau^{\prime} \geqq \tau$. In particular $\tau \in \mathfrak{I}$ for every $\tau \geqq 0$. It follows by the same argument as for $\mathfrak{L}_{0}$ that if $\tau_{0} \in \mathfrak{T}$ and $\mathfrak{B}_{\tau_{0}}$ denotes the set of points $z \in \mathfrak{L}_{\tau}, \tau>\tau_{0}$ then $a(z)-a\left(z_{0}\right)=s$ is a one to one conformal mapping of $\mathscr{S}_{\tau_{0}}$ onto the half plane

$$
s=\sigma+\tau i, \quad-\infty<\sigma<\infty, \quad \tau>\tau_{0} .
$$

In particular $\mathbb{S}_{\tau_{0}}$ is a simply connected domain, bounded from below by the simple Jordan curve $\mathfrak{L}_{r_{0}}$.

The linear set $\mathfrak{I}$ is open. For suppose that $\tau_{0} \in \mathfrak{I}$ and let $\sigma_{0}>0$ be as 
before, i.e. $\psi\left(\sigma+i \tau_{0}\right) \in \mathscr{D}\left(1, K_{0}\right)$ for $\sigma \geqq \sigma_{0} . b(z)$ is uniquely determined and holomorphic on $\mathfrak{I}_{\tau_{0}}$. Therefore for every $\sigma,-\sigma_{0} \leqq \sigma \leqq \sigma_{0}$, we can determine a positive number $\rho(\sigma)$ such that $b(z)$ is holomorphic and $b(z) \neq 0$ for $z-\left(\sigma+i \tau_{0}\right)<\rho(\sigma)$, and hence there is a positive number $t(\sigma)>0$ such that the equation

$$
\int_{\psi\left(\sigma+i \tau_{0}\right)}^{z} b(\zeta) d \zeta=\sigma+i \tau
$$

has a unique solution $z=\psi(\sigma+i \tau)$ for $\tau_{0}-t(\sigma) \leqq \tau \leqq \tau_{0}$.

In particular there is a $t_{0}>0$ such that $\psi(\sigma+i \tau)$ exists for $-\infty<\sigma<\infty$, $\tau_{0}-t_{0} \leqq \tau \leqq \tau_{0}$. For these $\tau, \mathfrak{L}_{\tau}$ is a regular iteration orbit since by the monodromy theorem, continuation of $b(z)$ along $\mathfrak{\Omega}_{\tau}$ from $z\left(\sigma_{0}+i \tau\right)$ to $z\left(-\sigma_{0}+i \tau\right)$ leads to the regular branch of $b(z)$ in $\mathfrak{D}\left(-1, K_{0}\right)$.

We now show that $\mathfrak{I}$ is a closed set. For suppose that $\mathfrak{L}_{\tau}$ is regular for every $t>\tau_{0}$. Let $n>2 \sigma_{0}+1, \sigma_{0} \leqq \sigma_{1} \leqq \sigma_{0}+1$, so that $-\sigma_{1}+n \geqq \sigma_{0}$. Then for

$$
\begin{aligned}
& \delta=\sigma_{0}+1-\sigma_{1}, 0 \leqq \delta \leqq 1, \psi\left(-\sigma_{0}-1+n+\delta+i \tau_{0}\right)=\psi\left(-\sigma_{1}+n+i \tau_{0}\right) \\
& =g_{n}\left(\psi\left(-\sigma_{1}+i \tau_{0}\right)\right) \\
& =\lim _{\tau \rightarrow r_{0}+} g_{n}\left(\psi\left(-\sigma_{1}+i \tau\right)\right) \\
& =\lim _{\tau \rightarrow \tau_{0}+} \psi\left(-\sigma_{1}+n+i \tau\right) \\
& =\lim _{\tau \rightarrow \tau_{0^{+}}} g_{d}\left(\psi\left(-\sigma_{0}-1+n+i \tau\right)\right) \\
& =g_{\delta}\left(\psi\left(-\sigma_{0}-1+n+i \tau_{0}\right)\right. \text {, }
\end{aligned}
$$

and $\mathfrak{\Omega}_{\tau_{0}}$ is regular.

Thus $\mathfrak{T}$ is both open and closed, hence identical with the whole real line and every $\mathfrak{R}_{\tau},-\infty<\tau<\infty$, is a regular iteration orbit.

If $\tau_{1}$ is as before, i.e. $\mathfrak{L}_{-\tau_{1}}$ bounds from above the domain $\mathbb{S}_{-\tau_{1}}^{*}$ consisting of all points $z \in \mathfrak{R}_{-r}, \tau>\tau_{1}$, we find that $\mathbb{S}_{-\tau_{1}} \cup \mathbb{S}_{-\tau_{1}}^{*} \cup \mathfrak{L}_{-\tau_{1}}$ is the whole complex plane and therefore $\mathscr{G}_{-\infty}=\lim _{r \rightarrow-\infty} \mathcal{G}_{r}$ is the whole complex plane. Thus $b(z)$ is finite and holomorphic over the whole plane and hence a constant. By property 2 of Lemma $1, b(z)=1, a(z)=z, g(z)=z+1$.

\section{The case $m>1$}

We shall briefly consider now the case when $m>1$ in (9). To apply the results of $\S 2$ it is convenient to transform $g(z)$ into

$$
g^{*}\left(z^{*}\right)=\frac{1}{m}(g(z))^{m}, \quad z^{*}=\frac{1}{m} z^{m},
$$

$g^{*}\left(z^{*}\right)$ has an algebraic expransion 


$$
g^{*}\left(z^{*}\right)=z^{*}+1+\sum_{k=1}^{\infty} a_{k}^{*} z^{*-k / m}
$$

at infinity and is meromorphic on the Riemann surface of $z^{* 1 / m}$ with the possible exception of the branchpoint $z^{*}=0$ where $g^{*}\left(z^{m}\right)$ may have an essential singularity.

A simple calculation gives the formal series

$$
\begin{aligned}
& g_{k}^{*}\left(z^{*}\right)=z^{*}+s+\sum_{k=1}^{\infty} a_{k}^{*}(s) z^{*-k / m}, \\
& b^{*}\left(z^{*}\right)=1-a_{1}^{*} z^{*-1 / m}+\sum_{k=2}^{\infty} b_{k}^{*} z^{*-k / m} .
\end{aligned}
$$

Lemma 1 remains valid for the various branches of $g^{*}\left(z^{*}\right)$, and we obtain from Lemma 4 , applied to the corresponding branches of $g_{\sigma}^{*}\left(z^{*}\right)$ :

THEOREM 2*. Suppose that each of the series $\left(10^{*}\right)$ converges for large $z^{*}$ when $s=\sigma,-1 \leqq \sigma \leqq 1$; then there exists an $R_{1}>0$ such that the series $\left(11^{*}\right)$ for $b^{*}\left(z^{*}\right)$ and each of the series $\left(10^{*}\right)$ for $g^{*}\left(z^{*}\right)(|s| \leqq 1)$ converges for $\left|z^{*}\right|>R_{1}$.

If $z_{0}^{*}, \sigma_{0}$ are as in (27) and (31) (with $z^{*}(\sigma), g_{\sigma}^{*}$ replacing $\left.z(\sigma), g_{\sigma}\right)$, we can define, by $(32),\left(32^{*}\right)$, an iteration orbit $\mathbb{R}_{\tau}^{*}$ for every real $\tau$ provided that $z^{*}(\sigma+i \tau) \neq 0$ or $\infty$ for any $\sigma$. If $\Omega_{\tau}^{*}$ passes through one of the branchpoints 0 or $\infty$, definition $\left(32^{*}\right)$ becomes ambiguous and some care is needed. It is best to transform the iteration orbits back to the $z$-plane where $z=\left(m z^{*}\right)^{1 / m}$.

Let $\mathfrak{L}_{\tau}$ denote the image of $\mathfrak{R}_{\tau}^{*}$ in the $z$-plane, with the specification that for $\sigma \leqq-\sigma_{0}, z(\sigma+i \tau) \in \mathbb{C}_{0}$ for every real $\tau$ where

$$
\mathfrak{E}_{0}=\left\{z=r e^{i \theta} \mid r>0, \pi / 2 m<\theta<3 \pi / 2 m\right\} .
$$

Then for $\boldsymbol{\tau} \geqq 0, \mathfrak{L}_{\boldsymbol{\tau}}$ is a regular iteration orbit situated in the sector

$$
\left\{z=r e^{i \theta} \mid r>0,0<\theta<\pi / m\right\}
$$

and satisfying

$$
\lim _{\sigma \rightarrow \infty} z(\sigma+i \tau)=\infty, \quad \lim _{\sigma \rightarrow \infty} z(-\sigma+i \tau)=\infty .
$$

Similarly for $\tau \leqq-\tau_{1}, \mathfrak{L}_{\boldsymbol{\tau}}$ is a regular iteration orbit situated in the sector

$$
\left\{z=r e^{i \theta} \mid r>0, \pi / m<\theta<2 \pi / m\right\}
$$

and satisfying $\left(29^{*}\right)$. The argument of $\S 3$ applies to the $\Omega_{\tau}$ (which, in the $z$-plane, are uniquely determined for every $\tau$ ), and we obtain the result that 


$$
b(z)=z^{m-1} b^{*}\left(\frac{1}{m} z^{m}\right)=z^{m-1}+\sum_{k=1}^{\infty} b_{k} z^{m-k-1}
$$

is holomorphic in the whole complex plane. Hence

$$
a(z)=\frac{1}{m} z^{m}+\sum_{k=1}^{m-1} c_{k} z^{m-k}
$$

is a polynomial of degree $m$. Therefore $w=g(z)$ where

$$
a(w)=a(z)+1
$$

is an algebraic function of degree $\leqq m$ and hence single valued only if it is rational. But for $m>1$ the equation (34) cannot have a rational $w=g(z)=p(z) / q(z)$ for solution as this would require $1 / m(p(z))^{m}$ to vanish at all zeros of $q(z)$. Therefore the conditions of Theorem $1^{*}$ cannot be satisfied when $m>1$.

\section{References}

[1] Baker, I. N., Zusammensetzungen ganzer Funktionen, Math. Z., 69 (1958), 121-163.

[2] Baker, I. N., Permutable power series and regular iteration, J. Australian Math. Soc., 2 (1962) 268-294.

The University of New South Wales,

Kensington, N.S.W. 